SLAC-PUB-8654

hep-ph/0010103

\title{
Experimental Probes of the Randall-Sundrum Infinite Extra Dimension
}

\author{
Daniel J. H. Chung* and Lisa Everett ${ }^{\dagger}$ \\ Randall Physics Laboratory, University of Michigan, Ann Arbor, Michigan 48109-1120 \\ Hooman Davoudiasl ${ }^{\ddagger}$ \\ Stanford Linear Accelerator Center, Stanford University, Stanford, CA 94309
}

\begin{abstract}
The phenomenological possibilities of the Randall-Sundrum non-compact extra dimension scenario with the AdS horizon increased to approximately a millimeter length, corresponding to an effective brane tension of $(\mathrm{TeV})^{4}$, are investigated. The corrections to the Newtonian potential are found to be the only observationally accessible probe of this scenario, as previously suggested in the literature. In particular, the presence of the continuum of KK modes does not lead to any observable collider signatures. The extent to which experimental tests of Newtonian gravity can distinguish this scenario from the scenario of Arkani-Hamed, Dimopoulos, and Dvali with one and two millimeter size extra dimensions is explicitly demonstrated.
\end{abstract}

*Electronic mail: djchung@umich.edu

${ }^{\dagger}$ Electronic mail: leverett@feynman.physics.lsa.umich.edu

†Electronic mail: hooman@slac.stanford.edu 
Much of the recent excitement and theoretical speculation regarding large/warped compact extra dimensions found for example in Refs. [1 - 4 has been fueled by the amelioration/novel rephrasing of the various hierarchy problems. In addition, the models of Refs. [1, 41] have raised some hopes that upcoming experiments may reveal signatures of the existence of higher dimensions [5 77]. On the other hand, the novelty of having phenomenologically consistent four-dimensional gravity in the presence of non-compact higher dimensions has been the main motivation for the investigations of "warped bulk" models based on the idea of Randall and Sundrum [8,9] without much motivation from upcoming experimental signature possibilities. In this paper, we investigate the possible experimental signatures of the model of Randall and Sundrum of Ref. [8] in the case that the warping scale is sufficiently lowered. Since most of the qualitative aspects of this scenario have already been discussed in Ref. [10] and Ref. [11], this paper will focus on the details of the experimental signatures.

Unlike the non-warped scenarios and some of the variations of the warped model for which the gravity is quasilocalized (see also [9, 12, 13, ), the original warped bulk model of Randall and Sundrum [8] (henceforth referred to as RS) did not seem to have any low energy phenomenological implications (perhaps with the exception of black hole physics [14]16,11]), mainly because the cosmological constant contribution coming from the constant energy density on the Planck brane had always been identified with the string scale for naive naturalness reasons. (We will refer to this constant energy density as the RS brane tension.) However, as explicitly noted for example by Kraus [17], if one attempts to identify the RS brane tension with that of a collection of D3 branes, one finds a discrepancy: the D3 brane tension is $2 / 3$ of the brane tension needed for the RS brane tension.

More recently, a different class of SUGRA solutions [18 (albeit singular) have been discovered which share the warped bulk spacetime of the RS scenario (although the noncompact limit has not been explored). Even more recently, there has also been progress in embedding the SUGRA containing the RS solution within a particular compactification of Type IIB string theory [19]. One emerging picture is that the RS brane does not correspond to any particular D-brane but is an "effective geometry" arising from a combination of a 
stack of negative tension branes stuck at an orbifold fixed point. Importantly, the positive quantity that was previously naively identified with the brane tension is not really the brane tension, but is actually a term arising from the combination of the positive curvature from the orbifold fixed point singularity and the negative tension of the branes confined there. In this picture, the RS brane tension is of the order of

$$
V_{\text {brane }} \sim M_{p l}^{2} k^{2}
$$

where $1 / k$ is the AdS horizon of the RS brane embedding spacetime (the warping scale). It is given by 17,19

$$
\frac{1}{k}=\frac{(4 \pi g N)^{1 / 4}}{M_{s t}}
$$

where $M_{s t}$ is the string scale, $g$ is the string coupling, and $2 N \gg 1$ is the number of D3-branes stacked to form the RS brane. Hence, if $N$ is taken large enough, the warping scale can be lowered to $k^{-1} \sim 0.1 \mathrm{~mm}$ such that the RS brane tension will be at $O(1 \mathrm{TeV})$, which may be a useful scale for model building (such as for particle/sparticle mass splitting). Additionally, given that the cosmological constant scale is $O(10 / \mathrm{mm})^{4}$, it may not be unreasonable to expect the $10 / \mathrm{mm}$ scale to enter the effective theory. In any case, the RS model with small warping will predict signatures for the upcoming experiments testing submillimeter behavior of Newton's law. We will refer to this model as the $\overline{\mathrm{RS}}$ model.円

As is well known, the leading order multiplicative correction to Newton's law has a functional behavior of $1+1 /\left(k^{2} r^{2}\right)$ for the RS model at large distances. Hence, one would naively expect this scenario to behave just like that of Ref. [1] (ADD model) with two extra dimensions $(n=2)$, which would imply that there would be collider signatures for the $\overline{\mathrm{RS}}$ model. On the other hand, as pointed out by Ref. [10], this $1+1 /\left(k^{2} r^{2}\right)$ correction becomes $1 /(k r)$ at distances much shorter than the AdS horizon length of $1 / k$. In other words, the

\footnotetext{
1 Although we restrict our investigations in this paper to the $\overline{\mathrm{RS}}$ model, our conclusions should easily extend to its generalizations such as that of Ref. [20].
} 
$\overline{\mathrm{RS}}$ model for $\sqrt{s} \gg k$ is effectively $\mathrm{ADD}(\mathrm{n}=1)$, since at large $\sqrt{s}$ the curvature can be ignored. Hence, at collider distances $(1 / \mathrm{TeV})$, for $k \sim(0.1 \mathrm{~mm})^{-1}$ the corrections from the continuum of KK states do not enhance the gravitational processes sufficiently to cause them to be observable.

However, it is not clear that there will be absolutely no effects in this case because the brane tension given by Eq. (11) is at the collider scale. It can be argued that since the RS brane is confined to an orbifold fixed point, the brane should not behave as a thick or "fat" branef even when the collider energy is above the scale of the brane tension. On the other hand, it is not obvious whether the stacking of such a large number of D-branes is consistent with the orbifold fixed point idealization. If the brane can fluctuate at the collider scale, the brane tension may serve as a cutoff for the standard model theory confined to the brane and may help solve the hierarchy problem (in analogy with the lowering of the fundamental scale in the ADD scenarios).

Even independently of the string theory picture (note that the string theory realization of the RS model is not known to be unique) and assuming somehow that a field theory can be well defined in the presence of orbifold fixed point singularities, the RS brane tension may not always reflect the scale of the tension of the object sitting at the orbifold fixed point. For example, suppose an extended object of tension $T_{b}$ is sitting at the orbifold fixed point but is not confined to the fixed point. Given that gravity itself is a derivative expansion of a more fundamental theory and that the curvature at the orbifold fixed point is singular, one would expect there to be large corrections to Einstein's equation arising from higher derivative curvature terms. Hence, one would find that the effective RS brane tension $T_{R S}$ is a sum of an infinite number of higher curvature terms and $T_{b}$ : e.g. schematically,

$$
T_{R S}=T_{b}+\alpha_{1} R^{2}+\alpha_{2} R^{3}\left(k / M^{3}\right)+\ldots,
$$

where the $R$ 's correspond to curvature quantities, the $\alpha_{i}$ correspond to coefficients of the

\footnotetext{
${ }^{2}$ For aspects of thick brane physics, see for example [21,6,22, 23.
} 
derivative expansion, and $M$ is the five-dimensional Planck scale.[1 Therefore, even if $T_{R S}=$ $O(\mathrm{TeV})$, one may in principle have a $T_{b}$ that is much larger, depending upon the precise nature of $\alpha_{i}$ and $R$. In that case, even without the "confining" effect of the orbifold fixed point, the brane would not "fatten" at a $\mathrm{TeV}$ scale. On the other hand, the brane may fatten such that there may be associated collider signatures and an amelioration of the hierarchy problem.

In this work, we assume that the RS brane can be treated as an idealized thin domain wall and neglect any possibility of brane fattening. As a partial follow-up to the work of Ref. [10], we investigate both the gravitational and collider experimental prospects for this scenario. We find that although there is no collider phenomenology, the corrections to Newton's law distinguish the $\overline{\mathrm{RS}}$ scenario from the ADD scenario. We find that not only the functional behavior of the gravitational correction is different but the magnitudes are sufficiently different to distinguish the two scenarios. Indeed, identifying the scale $R$ of the extra dimension in ADD with the AdS horizon length $1 / k$ of $\overline{\mathrm{RS}}$, we show that the $\overline{\mathrm{RS}}$ gravitational correction is much larger at the reach of the upcoming experiments. Owing to the larger number of moduli fields present in the the ADD scenario than in the $\overline{\mathrm{RS}}$ scenario, other signatures may distinguish the two scenarios. However, these more model dependent questions will not be addressed in this paper.

We first calculate the Newtonian potential generated by a point source of mass $m_{\text {source }}$ localized on the Planck brane. Explicitly, we consider the effects of the KK tower on the effective four-dimensional gravitational potential following the procedure of Garriga and Tanaka [15]. The details of this calculation can be found in Ref. [15], and several of the relevant results (including a derivation of the effective action to fix our notation and conventions) are listed in the Appendix. To determine for completeness the correction associated

\footnotetext{
${ }^{3}$ An investigation of domain wall solutions in the presence of higher derivative curvature terms can be found in Ref. [24].
} 
with an infrared cutoff in the AdS space, we assume the dimension transverse to the Planck brane is an $S^{1} / Z_{2}$ orbifold, and place a cutoff brane at the other orbifold fixed point. We then examine the limiting behavior as the distance $L$ between the orbifold fixed points is taken to infinity. In practice, it would of course be extremely difficult to stabilize a cutoff brane at macroscopic distances.' The potential can be written explicitly as a sum over terms involving Bessel functions:

$$
V=-G_{N} \frac{m_{\text {source }}}{r}\left[1+\frac{4}{3} \sum_{n=1} e^{-k a_{n} r}\left[\left(\frac{Y_{1}\left(a_{n}\right)}{Y_{1}\left(a_{n} e^{k L}\right)}\right)^{2}-1\right]^{-1}\right],
$$

in which $a_{n}=m_{n} / k \approx n \pi e^{-k L}$. However, for qualitative understanding it is important to note that the light $\mathrm{KK}$ modes (with $m_{n} \lesssim k$ ) couple to the matter on the brane with a strength $g_{c} \sim \sqrt{m_{n} / k}$ relative to the zero mode, while the heavy KK modes (with $m_{n} \gtrsim k$ ) couple with the same strength as the zero mode. The light states thus are responsible for the $1+1 /(k r)^{2}$ multiplicative correction to the Newtonian potential, while the heavy states provide a multiplicative correction of the form $1+1 /(k r)$. Hence there is a transition between the two regions, as first noted by Ref. [10]. To obtain a simple analytic expression for the potential, the two contributions must be patched together in the summation of the KK modes. We first interpolate between the two regions using a step-function approximation at the cutoff $q_{c} \sim O(k)$; we refer the reader to the Appendix for the details. We obtain

$$
\begin{aligned}
V & =-G_{N} \frac{m_{\text {source }}}{r}\left\{1+\frac{2}{3 k^{2} r^{2}}-e^{-q_{c} r}\left[\frac{2}{3 k^{2} r^{2}}+\frac{2}{3\left(\frac{k}{q_{c}}\right) k r}-\frac{4}{3 \pi k r}+\left(\frac{2}{3}-\frac{\pi}{3\left(\frac{k}{q_{c}}\right)}\right) e^{-k L}\right]\right. \\
& \left.-\frac{1}{k r} \frac{4}{3 \pi} e^{-M r}+\ldots\right\}
\end{aligned}
$$

where $G_{N}$ is the four-dimensional Newton's constant, and we have kept only the leading terms in $e^{-k L}$ suppression. Note that terms that depend explicitly on the "light mode-

\footnotetext{
${ }^{4}$ Furthermore, arranging a stabilization mechanism for the radion would also obviate one of the original motivations for having the warp factor "truncate" the volume of the extra dimension because in some sense the brane tension in the RS model is meant to replace the role of the bulk stabilizing potential.
} 
cutoff" $q_{c}$ in Eq. (5) are sensitive to our step function treatment of the gravitational coupling between the states with $m_{n} \lesssim q_{c}$ and $m_{n} \gtrsim q_{c}$. Note in particular that since $e^{-q_{c} r}$ is sensitive to the value of $q_{c}$, we expect the exact form of this exponential to be an artifact of the abrupt change in the coupling $g_{c}$ of the KK states between $m_{n} \lesssim q_{c}$ and $m_{n} \gtrsim q_{c}$ to the matter on the brane. An argument for there being no exponential suppression is presented in Ref. [25].

Note that even in the $L \rightarrow \infty$ limit, the $e^{-q_{c} r} / k r$ term survives. Presumably, in the original calculation of $\mathrm{RS}$, this term was neglected due to the fact that $k$ was near the Planck scale. Note that in the limit that $k r \rightarrow 0$ with $q_{c}=O(1) k$,

$$
V=-G_{N} \frac{m_{\text {source }}}{r}\left[1+\frac{4}{3 \pi} \frac{1}{k r}\left(1-e^{-M r}\right)+\frac{1}{3\left(\frac{k}{q_{c}}\right)^{2}}-\frac{4}{3\left(\frac{k}{q_{c}}\right) \pi}+O(k r)\right]
$$

showing that the theory becomes five dimensional.f Hence, as pointed out by Ref. [10], the behavior for the Newtonian potential makes a smooth transition between two and one extra flat dimensional behavior. As we shall see, this is consistent with the consideration in the momentum space applicable for colliders.

One can obtain a perhaps more accurate approximate expression for the potential using the approximation

$$
Y_{1}^{2}\left(a_{n} e^{k L}\right) \approx \frac{2 e^{-k L}}{\pi a_{n}} \frac{Y_{1}^{2}\left(a_{n}\right)}{J_{1}^{2}\left(a_{n}\right)+Y_{1}^{2}\left(a_{n}\right)}
$$

This formula can be obtained by approximating $J_{1}\left(a_{n} e^{k L}\right)$ and $Y_{1}\left(a_{n} e^{k L}\right)$ as the leading sinusoidal function. After taking the $k L \rightarrow \infty$ limit, this results in

$$
V=-G_{N} \frac{m_{\text {source }}}{r}\left[1+\frac{8}{3 \pi^{2}} \int_{0}^{\infty} \frac{d a}{a} \frac{e^{-a k r}}{J_{1}^{2}(a)+Y_{1}^{2}(a)}\right]
$$

which is particularly useful for evaluating the Newtonian potential corrections numerically. Note that unlike Eq. (5), there is no artificial separation between the light $\left(m_{n} \lesssim k\right)$ and heavy $\left(m_{n} \gtrsim k\right)$ KK states. Eq. (5) matches Eq. (8) most closely when $q_{c}=2 k / \pi$. The ratio

\footnotetext{
${ }^{5}$ Note that Eq. (6) agrees with Eq. (3.38) of [16] in the $k r \ll 1$ limit.
} 


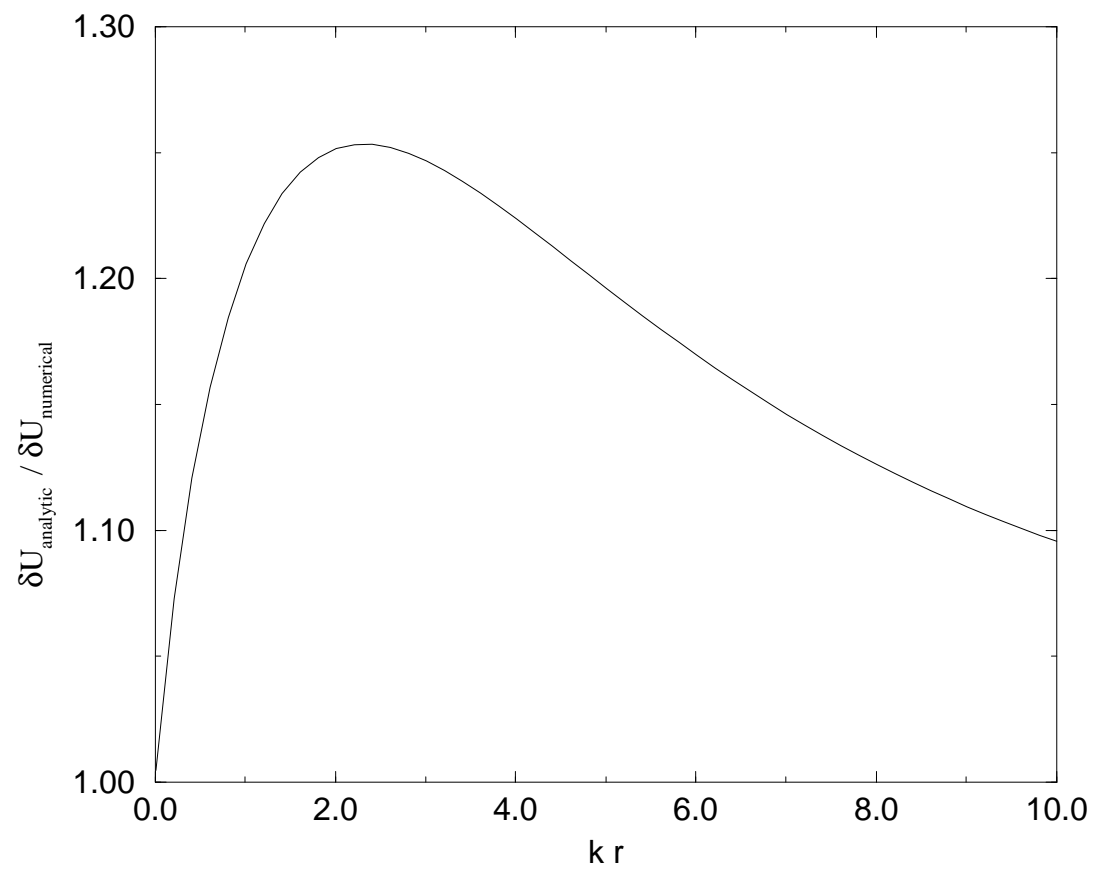

FIG. 1. A comparison of (8) with (5) is given. The Newtonian potential is given by $-\left(G_{N} m_{\text {source }} / r\right)\left(1+\delta U_{X}\right)$ where $\delta U_{\text {analytic }}$ is given by Eq. (5) and $\delta U_{\text {numerical }}$ is given by Eq. (8).

of the numerical values of the correction $\delta U$ (where $\delta U$ is given by $V=\left(G_{N} m_{\text {source }} / r\right)[1+$ $\delta U])$ of the two equations are shown in Fig.1.

Let us now compare this with the predictions of the ADD model for two extra dimensions. Systematic corrections to the Newtonian potential have been worked out explicitly in [26,27]; we use the results of Ref. [27] and list the relevant expressions in Table [ under the heading "ADD." In the table, we take $q_{c}=2 k / \pi$ since that gives the closest match to the numerical approximation of the correction. We have assumed that the two extra dimensions of the ADD scenario each has a length of $2 \pi R$. Note that the small $r / R$ expansion can be obtained through the method of images. It is interesting to note that there is no $R / r$ correction in this potential, even though an integration approximation of the summation would suggest that there be one.

In Fig. 2 we see the comparison between the $\overline{\mathrm{RS}}$ and the ADD scenarios for $R=1 / k$. In 


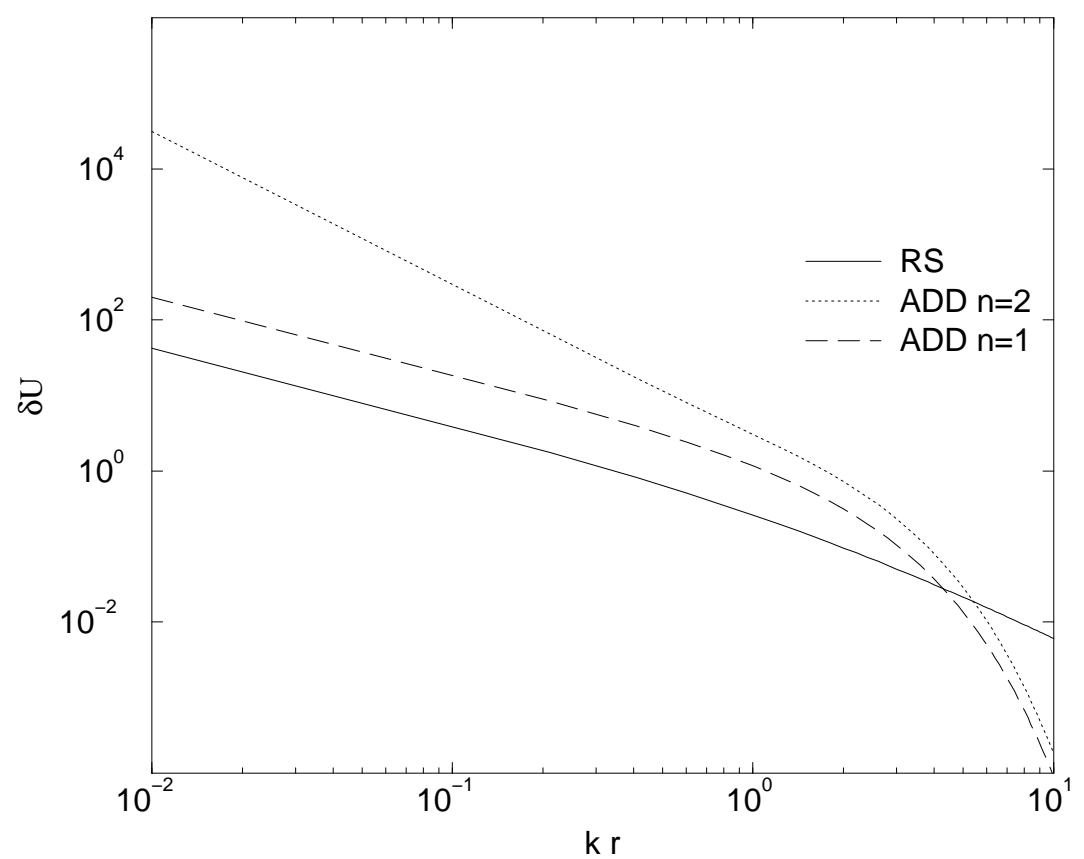

FIG. 2. The corrections to the Newtonian potential are plotted as a function of a scaled radius for the ADD scenario with one and two extra dimensions and the $\overline{\mathrm{RS}}$ scenario. The radius $R$ of the ADD scenario is identified with the AdS horizon $1 / k$.

addition to the two-extra-dimension scenario which is well motivated from the point of view of the hierarchy problem, we will also consider the one extra dimension ADD scenario with the compactification scale $R$ set to $1 / k$, corresponding to a five dimensional Planck scale of $10^{8} \mathrm{GeV}$. Specifically, we plot $\delta U$ for the ADD model with two extra dimensions $(n=2)$ and with one extra dimension $(n=1)$. For the two extra dimensions case, we use

$$
\delta U=2 \sum_{n_{1}, n_{2}} e^{-\sqrt{n_{1}^{2}+n_{2}^{2}} \frac{r}{R}}
$$

while for the one extra dimension case

$$
\delta U=\frac{2}{e^{r / R}-1}
$$

The figure compares these to the $\delta U$ for the $\overline{\mathrm{RS}}$ case given by Eq. (8). It shows that for $k \sim R^{-1} \sim 10(\mathrm{~mm})^{-1}$ and $r=1 \mathrm{~mm}$, multiplicative corrections of $1+O\left(10^{-2}\right)$ arise from the $\overline{\mathrm{RS}}$ scenario which may be measured in the upcoming set of experiments (for a recent 
review, see Ref. [28]) while the corrections arising from the ADD scenarios are too small to be detected. Therefore, the ADD and $\overline{\mathrm{RS}}$ scenarios can be clearly distinguished both at short and long distances (compared to the "compactification scale" $1 / k$ ) through not only the functional behavior but also the amplitudes.

Let us now consider the collider signatures of the $\overline{\mathrm{RS}}$ model. For a typical graviton mediated $s$-channel scattering process in $\overline{\mathrm{RS}}$ at the center of mass energy $\sqrt{s} \gg k$, we have for the scattering amplitude $A$

$$
A \sim s^{2} \sum_{n} \frac{i g_{c}^{2}(n)}{s-m_{n}^{2}}
$$

where we have explicitly denoted the $n$ dependence of $g_{c}$. As in the gravitational potential section, we take the UV cutoff to be at the 5-D Planck scale $M$, at which we expect a more fundamental theory to take over. Since we are making order of magnitude estimates, we can approximate the summation as an integral, and divide the KK states into the light $\left(m_{n} \lesssim k\right)$ and heavy $\left(m_{n} \gtrsim k\right)$ groups as done in the calculation of the Newtonian potential. We find

$$
A \sim \frac{s}{M_{p l}^{2}}\left[\frac{8 \pi \sqrt{s}}{k}+i \frac{16 s}{k M}\right],
$$

where we have used the approximations $k \ll \sqrt{s}, k L \gg 1, \sqrt{s} \ll M$. Hence the values $s \sim 10^{6} \mathrm{GeV}^{2}$ and $k \sim 10^{-12} \mathrm{GeV}$ relevant for collider experiments imply $A \sim\left(10^{-14}+i 10^{-19}\right)$ where the real part corresponds to the resonance channel. The easiest way to see that this number is too small for observable collider phenomenology is to compare this to the amplitude obtained in the ADD scenario with two extra dimensions. With the cutoff scale at $\Lambda=10 \mathrm{TeV}$ in the ADD scenario, the amplitude is

$$
A_{A D D} \sim \frac{-i s^{2}}{\Lambda^{4}} \ln \left(\frac{\Lambda^{2}}{s}\right) \sim-i 10^{-4} .
$$

Hence, even with the resonance enhancement of $A$ taken into account, there is no observable phenomenology for the RS scenario even with $k$ as small as $10^{-3} \mathrm{eV}$.

\footnotetext{
${ }^{6}$ Similarly, there will be no collider signatures for the one extra dimension ADD model with $R \sim 0.1 \mathrm{~mm}$.
} 
In summary, we considered the uncompactified RS model of localized gravity in the limit where the warp scale $k$ is small, the $\overline{\mathrm{RS}}$ model, and studied its possible experimental consequences. We showed that for $k \sim 10^{-3} \mathrm{eV}$, this model could have measurable contributions to deviations from Newtonian gravity, at near future levels of experimental sensitivity. Naturalness arguments may favor $k$ to be of order the fundamental scale. However, a realistic fundamental theory of quantum gravity is as yet unknown, and the only candidate, string theory, does not yield any single RS picture. Hence, without any rigorous requirements at hand, we treat $k$ as a model parameter. Nonetheless, we point out that this low value of $k$ yields an effective energy scale of $O(\mathrm{TeV})$ for the four-dimensional boundary corresponding to our visible universe, a scale which may be useful for resolving the hierarchy problem. This leaves open the possibility that perhaps in some more fundamental picture, low energy supersymmetry breaking or other weak scale physics may require an effective $k \sim 10^{-3} \mathrm{eV}$.

We studied the contribution of the graviton KK modes to the four-dimensional gravitational potential of a source mass, resulting in a deviation $\delta U$ from Newtonian gravity. We not only verified the $k r \gg 1$ and $k r \ll 1$ behavior first argued by Ref. [10], but also obtained expressions for the intermediate regime. We found that for $k r \gtrsim 1$, the $\overline{\mathrm{RS}}$ model results in values of $\delta U$ that are about two orders of magnitude larger than the corresponding ADD values for one and two extra dimensions of size $R \sim k^{-1}$. This makes the $\overline{\mathrm{RS}}$ model distinguishable from the ADD scenarios in the near future gravitational experiments. We

finally noted that the $\overline{\mathrm{RS}}$ model does not yield collider signatures for $\sqrt{s} \gg k \sim(0.1 \mathrm{~mm})^{-1}$. In this regime, the theory is basically that of ADD with one extra dimension of tenth of a millimeter size and with a fundamental scale $M \sim 10^{8} \mathrm{GeV}$, which is far beyond the reach of present and foreseeable colliders.

\section{ACKNOWLEDGMENTS}

It is a pleasure to thank J. Liu for conversations regarding his papers and M. Peskin for discussions regarding the corrections to Newton's Law. We gratefully acknowledge conver- 
sations with K. Choi, S. Kachru, J. Lykken, S. Rigolin, T. Rizzo, M. Schmaltz, R. Sundrum, D. Waldram, and J. Wang. Finally, we thank the Aspen Center for Physics, where this work was originated, for hospitality. The work of D.J.H.C. and L.E. was supported in part by the Department of Energy. The work of H.D. is supported by the Department of Energy, contract DE-AC03-76SF00515. 


\section{REFERENCES}

[1] N. Arkani-Hamed, S. Dimopoulos and G. Dvali, "The hierarchy problem and new dimensions at a millimeter," Phys. Lett. B429, 263 (1998) hep-ph/9803315.

[2] I. Antoniadis, "A Possible New Dimension At A Few Tev," Phys. Lett. B246, 377 (1990).

[3] K. R. Dienes, E. Dudas and T. Gherghetta, "Extra spacetime dimensions and unification," Phys. Lett. B436, 55 (1998) hep-ph/9803466.

[4] L. Randall and R. Sundrum, "A large mass hierarchy from a small extra dimension," Phys. Rev. Lett. 83, 3370 (1999) hep-ph/9905221.

[5] N. Arkani-Hamed, S. Dimopoulos and G. Dvali, "Phenomenology, astrophysics and cosmology of theories with sub-millimeter dimensions and $\mathrm{TeV}$ scale quantum gravity," Phys. Rev. D59, 086004 (1999) hep-ph/9807344;I. Antoniadis, N. Arkani-Hamed, S. Dimopoulos and G. Dvali, "New dimensions at a millimeter to a Fermi and superstrings at a TeV," Phys. Lett. B436, 257 (1998) hep-ph/9804398; G. F. Giudice, R. Rattazzi and J. D. Wells, "Quantum gravity and extra dimensions at high-energy colliders," Nucl. Phys. B544, 3 (1999) hep-ph/9811291]; S. Nussinov and R. Shrock, "Some remarks on theories with large compact dimensions and TeV-scale quantum gravity," Phys. Rev. D59, 105002 (1999) hep-ph/9811323; E. A. Mirabelli, M. Perelstein and M. E. Peskin, "Collider signatures of new large space dimensions," Phys. Rev. Lett. 82, 2236 (1999) [hep-ph/9811337]; T. Han, J. D. Lykken and R. Zhang, "On Kaluza-Klein states from large extra dimensions," Phys. Rev. D59, 105006 (1999) hep-ph/9811350];J. L. Hewett, "Indirect collider signals for extra dimensions," Phys. Rev. Lett. 82, 4765 (1999) hep-ph/9811356]; G. Shiu, R. Shrock and S. H. Tye, "Collider signatures from the brane world," Phys. Lett. B458, 274 (1999) hep-ph/9904262; S. Cullen and M. Perelstein, "SN1987A constraints on large compact dimensions," Phys. Rev. Lett. 83, 268 (1999) hep-ph/9903422]; L. J. Hall and D. Smith, "Cosmological con- 
straints on theories with large extra dimensions," Phys. Rev. D60, 085008 (1999) hepph/9904267]; V. Barger, T. Han, C. Kao and R. J. Zhang, "Astrophysical constraints on large extra dimensions," Phys. Lett. B461, 34 (1999) hep-ph/9905474; C. Hanhart, D. R. Phillips, S. Reddy and M. J. Savage, "Extra dimensions, SN1987a, and nucleon nucleon scattering data," nucl-th/0007016; P. Binetruy and J. Silk, "Probing large-distance higher-dimensional gravity with cosmic microwave background measurements," astro-ph/0007452; P. Binetruy, C. Deffayet and D. Langlois, "Non-conventional cosmology from a brane-universe," Nucl. Phys. B565, 269 (2000) hep-th/9905012; D. J. Chung and K. Freese, "Cosmological challenges in theories with extra dimensions and remarks on the horizon problem," Phys. Rev. D61, 023511 (2000) hep-ph/9906542; D. J. Chung, E. W. Kolb and A. Riotto, "Extra dimensions present a new flatness problem," hep-ph/0008126.

[6] N. Arkani-Hamed and M. Schmaltz, "Hierarchies without symmetries from extra dimensions," Phys. Rev. D61, 033005 (2000) hep-ph/9903417; N. Arkani-Hamed, Y. Grossman and M. Schmaltz, "Split fermions in extra dimensions and exponentially small cross-sections at future colliders," hep-ph/9909411.

[7] H. Davoudiasl, J. L. Hewett and T. G. Rizzo, "Phenomenology of the Randall-Sundrum gauge hierarchy model," Phys. Rev. Lett. 84, 2080 (2000) hep-ph/9909255; H. Davoudiasl, J. L. Hewett and T. G. Rizzo, "Bulk gauge fields in the Randall-Sundrum model," Phys. Lett. B473, 43 (2000) hep-ph/9911262 ; H. Davoudiasl, J. L. Hewett and T. G. Rizzo, "Experimental probes of localized gravity: On and off the wall," hepph/0006041; H. Davoudiasl, J. L. Hewett and T. G. Rizzo, "The (g-2) of the muon in localized gravity models," hep-ph/0006097.

[8] L. Randall and R. Sundrum, "An alternative to compactification," Phys. Rev. Lett. 83, 4690 (1999) [hep-th/9906064].

[9] I. I. Kogan, S. Mouslopoulos, A. Papazoglou, G. G. Ross and J. Santiago, "A three three- 
brane universe: New phenomenology for the new millennium?," Nucl. Phys. B584, 313 (2000) hep-ph/9912552]; R. Gregory, V. A. Rubakov and S. M. Sibiryakov, "Opening up extra dimensions at ultra-large scales," Phys. Rev. Lett. 84, 5928 (2000) thepth/0002072]; J. Lykken, R. C. Myers and J. Wang, "Gravity in a box," JHEP 0009, 009 (2000) hep-th/0006191.

[10] N. Arkani-Hamed, S. Dimopoulos, G. Dvali and N. Kaloper, "Infinitely large new dimensions," Phys. Rev. Lett. 84, 586 (2000) hep-th/9907209].

[11] S. B. Giddings and E. Katz, "Effective theories and black hole production in warped compactifications," hep-th/0009176.

[12] J. Lykken and L. Randall, "The shape of gravity," JHEP 0006, 014 (2000) hepth/9908076].

[13] P. Binetruy and J. Silk, "Probing large-distance higher-dimensional gravity with cosmic microwave background measurements," astro-ph/0007452.

[14] R. Emparan, G. T. Horowitz and R. C. Myers, "Exact description of black holes on branes. II: Comparison with BTZ black holes and black strings," JHEP 0001, 021 (2000) hep-th/9912135; R. Emparan, G. T. Horowitz and R. C. Myers, "Exact description of black holes on branes," JHEP 0001, 007 (2000) hep-th/9911043.

[15] J. Garriga and T. Tanaka, "Gravity in the brane-world," Phys. Rev. Lett. 84, 2778 (2000) hep-th/9911055.

[16] S. B. Giddings, E. Katz and L. Randall, "Linearized gravity in brane backgrounds," JHEP 0003, 023 (2000) hep-th/0002091.

[17] P. Kraus, "Dynamics of anti-de Sitter domain walls," JHEP 9912, 011 (1999) thepth/9910149].

[18] A. Falkowski, Z. Lalak and S. Pokorski, "Supersymmetrizing branes with bulk in five- 
dimensional supergravity," hep-th/0004093; T. Gherghetta and A. Pomarol, "Bulk fields and supersymmetry in a slice of AdS," hep-ph/0003129; R. Altendorfer, J. Bagger and D. Nemeschansky, "Supersymmetric Randall-Sundrum scenario," hep-th/0003117.

[19] E. Bergshoeff, R. Kallosh and A. Van Proeyen, "Supersymmetry in singular spaces," hep-th/0007044; M. J. Duff, J. T. Liu and W. A. Sabra, "Localization of supergravity on the brane," hep-th/0009212; J. T. Liu and H. Sati, "Breathing mode compactifications and supersymmetry of the brane-world," hep-th/0009184; M. J. Duff and J. T. Liu, "On the equivalence of the Maldacena and Randall-Sundrum pictures," Phys. Rev. Lett. 85, 2052 (2000) hep-th/0003237.

[20] T. Gherghetta, E. Roessl and M. Shaposhnikov, "Living inside a hedgehog: Higherdimensional solutions that localize gravity," hep-th/0006251.

[21] C. Csaki, J. Erlich, T. J. Hollowood and Y. Shirman, "Universal aspects of gravity localized on thick branes," Nucl. Phys. B581, 309 (2000) hep-th/0001033; M. Gremm, "Thick domain walls and singular spaces," Phys. Rev. D62, 044017 (2000) hepth/0002040.

[22] A. De Rujula, A. Donini, M. B. Gavela and S. Rigolin, "Fat brane phenomena," Phys. Lett. B482, 195 (2000) hep-ph/0001335.

[23] H. Georgi, A. Grant and G. Hailu, "Chiral fermions, orbifolds, scalars and fat branes," hep-ph/0007350.

[24] O. Corradini and Z. Kakushadze, "Localized gravity and higher curvature terms," hepth/0009022.

[25] R. Dick and D. Mikulovicz, "Gravity and the Newtonian limit in the Randall-Sundrum model," Phys. Lett. B476, 363 (2000) hep-th/0001013].

[26] A. Kehagias and K. Sfetsos, "Deviations from the $1 / \mathrm{r}^{* *} 2$ Newton law due to extra dimensions and alpha' corrections," Phys. Lett. B472, 39 (2000) hep-ph/9905417. 
[27] E. G. Floratos and G. K. Leontaris, "Low scale unification, Newton's law and extra dimensions," Phys. Lett. B465, 95 (1999) hep-ph/9906238.

[28] J. C. Long, H. W. Chan and J. C. Price, "Experimental status of gravitational-strength forces in the sub-centimeter regime," Nucl. Phys. B539, 23 (1999) hep-ph/9805217.

\section{APPENDIX A: EFFECTIVE ACTION}

To fix our notation, let us review the construction of the four-dimensional effective action presented in Ref. [8]. Consider the perturbed metric

$$
d s^{2}=f(z)\left[\eta_{\mu \nu}+h_{\mu \nu}(x)\right] d x^{\mu} d x^{\nu}-d z^{2},
$$

where the transverse dimension is parameterized by $z$ (the Planck brane is located at $z=0$ ), and the warp factor is

$$
f(z)=e^{-2 k|z|} .
$$

We then find the effective Lagrangian for the metric perturbation to be

$$
\Delta[\sqrt{g}(R+2 \Lambda)]=-\frac{f}{2}\left[\frac{-1}{2} h^{\mu \nu} h_{\mu \nu, \alpha}^{, \alpha}+h^{\mu \nu} h_{\nu, \lambda \mu}^{\lambda}-h^{\mu \nu} h_{, \mu \nu}+\frac{1}{2} h h_{, \mu}^{, \mu}\right]+\frac{f^{2}}{4}\left[h^{\mu \nu}{ }_{, 4} h_{\mu \nu, 4}-h_{, 4}^{2}\right]
$$

where $R$ is the five-dimensional Ricci scalar, $\Lambda$ is the bulk cosmological constant, and the indices (which are contracted with the Minkowski metric $\eta_{\mu \nu}$ ) run over $0,1,2,3$, the coordinates parallel to the Planck brane. In the transverse, traceless gauge, we simply have

$$
\Delta[\sqrt{g}(R+2 \Lambda)]=\frac{-f}{4} h^{\mu \nu} h_{\mu \nu, \alpha}^{, \alpha}
$$

Hence, the graviton action $S_{G}$ coupled minimally to the matter action $S_{M}$ is given by

$$
S=S_{G}+S_{M}
$$

where 


$$
S_{G}=\frac{M^{3}}{4} \int d^{5} x f h^{\mu \nu} h_{\mu \nu, \alpha}^{, \alpha}
$$

Let us decompose the graviton in terms of 4-dimensional modes:

$$
h_{\mu \nu}=\xi \sqrt{\frac{\pi}{L}} \sum_{n} H_{\mu \nu}^{(n)} \chi^{(n)}
$$

in which

$$
\begin{gathered}
\partial_{z}\left(f^{2} \partial_{z} \chi^{(n)}\right)+m_{n}^{2} f(z) \chi^{(n)}=0 \\
\partial_{z} \chi^{(n)}(0)=\partial_{z} \chi^{(n)}(L)=0
\end{gathered}
$$

with the normalization

$$
\int_{-L}^{L} d z f(z) \chi^{(n)}(z) \chi^{(m)}(z)=\frac{L}{\pi} \delta_{n m}
$$

Note that $\xi$ is an arbitrary normalization parameter. Hence, we find for the four dimensional effective action of gravity

$$
S_{G}=\frac{\xi^{2} M^{3}}{4} \int d^{4} x\left\{H_{(n)}^{\mu \nu} H_{\mu \nu, \alpha}^{(n), \alpha}+m_{n}^{2} H_{(n)}^{\mu \nu} H_{\mu \nu}^{(n)}\right\}
$$

We can also expand the matter action to give

$$
\begin{aligned}
S_{M} & =S_{M}^{(0)}+\int d^{4} x \frac{\delta S}{\delta g_{\mu \nu}(x)} h_{\mu \nu}(x, z=0)+\ldots \\
& =S_{M}^{(0)}-\sum_{n} \int d^{4} x \frac{\sqrt{g(z=0)}}{2} T_{\text {brane }}^{\mu \nu} H_{\mu \nu}^{(n)} \chi^{(n)}(z=0) \xi \sqrt{\frac{\pi}{L}}+\ldots
\end{aligned}
$$

where the ... include comparable coupling terms induced from the fact that the brane is bent in the presence of matter in the transverse traceless gauge. The effective action is given by

$$
\delta S=\frac{\xi^{2} M^{3}}{4} \int d^{4} x\left\{H_{(n)}^{\mu \nu} H_{\mu \nu, \alpha}^{(n), \alpha}+m_{n}^{2} H_{(n)}^{\mu \nu} H_{\mu \nu}^{(n)}\right\}-\frac{1}{2} \int d^{4} x T_{b r a n e}^{\mu \nu} H_{\mu \nu}^{(n)} \chi^{(n)}(z=0) \xi \sqrt{\frac{\pi}{L}}+\ldots
$$


To see the Planck scale suppression of the couplings, it is convenient to normalize the kinetic term for the gravitons such that $H_{\mu \nu}$ has dimension 1 and the propagator resembles a scalar propagator. Hence, we let

$$
\xi^{2}=\frac{4}{M^{3}}
$$

Now, we have for $z>0$,

$$
\chi^{(n)}(z)=\frac{1}{N_{n}}\left[J_{2}\left(a_{n} e^{k z}\right)+\alpha_{n} Y_{2}\left(a_{n} e^{k z}\right)\right] e^{2 k z}
$$

with

$$
\alpha_{n}=\frac{-J_{1}\left(a_{n}\right)}{Y_{1}\left(a_{n}\right)}
$$

where

$$
a_{n}=\frac{m_{n}}{k}
$$

and the mass condition is given by

$$
\frac{J_{1}\left(a_{n} e^{k L}\right)}{J_{1}\left(a_{n}\right)}=\frac{Y_{1}\left(a_{n} e^{k L}\right)}{Y_{1}\left(a_{n}\right)} .
$$

To get the normalization constant $N_{n}$, we use

$$
\frac{2}{N_{n}^{2}} \int_{0}^{L} d z\left[J_{2}\left(a_{n} e^{k z}\right)+\alpha_{n} Y_{2}\left(a_{n} e^{k z}\right)\right]^{2} e^{2 k z}=\frac{L}{\pi} .
$$

Writing

$$
Z_{n}(x) \equiv J_{n}(x)+\alpha Y_{n}(x)
$$

for any constant $\alpha$ and real argument $x$, we can use the Bessel function identity

$$
\int d x x Z_{n}^{2}(a x)=\frac{x^{2}}{2}\left[Z_{n}^{2}(a x)-Z_{n+1}(a x) Z_{n-1}(a x)\right]
$$

for any integer $n$ and the mass shell condition to get

$$
N_{n}^{2}=\frac{\pi}{k L}\left[e^{2 k L} Z_{2}^{2}\left(a_{n} e^{k L}\right)-Z_{2}^{2}\left(a_{n}\right)\right] .
$$


Therefore, the factor $\chi^{(n)}$ which determines the matter coupling to gravity is given by

$$
\chi^{(n)}(0)=\sqrt{\frac{k L}{\pi}} \frac{1}{\sqrt{\left[\frac{Y_{1}\left(a_{n}\right)}{Y_{1}\left(a_{n} e^{k L}\right)}\right]^{2}-1}}
$$

in which we used the Bessel function identity

$$
J_{\nu}(x) Y_{\nu+1}(x)-J_{\nu+1}(x) Y_{\nu}(x)=-\frac{2}{\pi x},
$$

and the definition of $\alpha_{n}$. The coupling of the gravitational modes to matter is then given by

$$
\delta S_{M}=\int d^{4} x T^{\mu \nu} g_{c}^{(n)} H_{\mu \nu}^{(n)},
$$

where we have defined the coupling

$$
g_{c}^{(n)} \equiv \frac{-\xi}{2} \sqrt{\frac{\pi}{L}} \chi^{(n)}(z=0)=\frac{-1}{M^{3 / 2}} \sqrt{\frac{\pi}{L}} \chi^{(n)}(z=0) .
$$

\section{APPENDIX B: NEWTONIAN POTENTIAL}

We now calculate the Newtonian potential generated by a point source of mass $m_{\text {source }}$ localized on the brane, following the procedure of Garriga and Tanaka, [15]. We find

$$
V=-\frac{1}{2 M^{3}} m_{\text {source }}\left\{\frac{2}{3} \sum_{n} \frac{e^{-m_{n} r}}{4 \pi r} \frac{\pi \chi_{n}^{2}(0)}{L}-\frac{k}{24 \pi r}\right\}
$$

where the factor of $2 / 3$ can be attributed to the tensorial character of gravity and brane bending. Note that since the bound state mode $\chi_{0}$ is normalized as

$$
\chi_{0}=\sqrt{\frac{k L}{\pi\left(1-e^{-2 k L}\right)}},
$$

the recovery of Newton's law for this mode requires

$$
\frac{M_{p l}^{2}}{8 \pi}=\frac{2 M^{3}}{k}
$$

to first order in $e^{-k L}$ (where $M_{p l}^{2} \equiv 1 / G_{N}$ ). Hence, we have

$$
g_{c}=-\frac{\sqrt{2}}{\left(M_{p l} / \sqrt{8 \pi}\right)} \frac{1}{\sqrt{\left[\frac{Y_{1}\left(a_{n}\right)}{Y_{1}\left(a_{n} e^{k L}\right)}\right]^{2}-1}}
$$

Let us examine $g_{c}$ in various limits, 
1. $a_{n} \ll 1$ : In this caset

$$
g_{c}=-\frac{2 \pi \sqrt{2}}{M_{p l}} \sqrt{\frac{m_{n}}{k}} e^{-\frac{k L}{2}}+O\left(e^{-3 k L}\right)
$$

2. $a_{n} \gg 1$ : In this case

$$
g_{c}=-\frac{4 \sqrt{\pi}}{M_{p l}}\left\{e^{-\frac{k L}{2}}\left[1-\frac{15}{128}\left(\frac{k}{m_{n}}\right)^{2}\right]+e^{-\frac{3 k L}{2}}\left[\frac{1}{2}-\frac{45}{256}\left(\frac{k}{m_{n}}\right)^{2}\right]+O\left(e^{-\frac{5 k L}{2}}\right)\right\}
$$

where we have kept the leading term correction in $e^{-k L}<1$ suppression. For both cases it is valid to approximate $a_{n} \approx[n+1 / 4-\epsilon(n)] \pi e^{-k L}$ where $\epsilon(n) \approx 3 /\left[8 \pi^{2}(n+1 / 4)\right]$. In the expressions above, and from now on, we neglect all dependence on $\epsilon(n)$. 9 Note that the $\left(k / m_{n}\right)^{2}$ terms in Eq. (B6) can also be neglected.

In summing over the KK states, we split the states into two groups, one with $m_{n} \leq q_{c}$ $\left(q_{c}\right.$ is a scale with order of magnitude $O\left(q_{c}\right)=k$ ) and coupling Eq. (B5), and the other with $m_{n}>q_{c}$ and coupling Eq. (B6). In reality, there is some smooth function approximated by Eq. (B4) that should be used instead, but for the purposes of carrying out the summation explicitly, we have used Eq. (B5) for modes $n \leq-1 / 4+q_{c} e^{k L} / k \pi$ and Eq. (B6) for the rest (essentially a step function treatment). We have also taken the cutoff for the $m_{n}>q_{c}$ modes to be $M$ (i.e. restricted to modes $\left.n<-1 / 4+M e^{k L} / k \pi\right)$. Carrying out the summation (without using an integral approximation), we obtain Eq. (5)

$$
\begin{aligned}
V & =-\frac{1}{M_{p l}^{2}} \frac{m_{\text {source }}}{r}\left\{1+\frac{2}{3 k^{2} r^{2}}-e^{-q_{c} r}\left[\frac{2}{3 k^{2} r^{2}}+\frac{2}{3\left(\frac{k}{q_{c}}\right) k r}-\frac{4}{3 \pi k r}+\left(\frac{2}{3}-\frac{\pi}{3\left(\frac{k}{q_{c}}\right)}\right) e^{-k L}\right]\right. \\
& \left.-\frac{1}{k r}\left(\frac{4}{3 \pi} e^{-M r}\right)+\ldots\right\},
\end{aligned}
$$

where we have kept terms to only leading order in $O\left(e^{-k L}\right)$ and $O\left(e^{-M r}\right)$.

\footnotetext{
${ }^{7}$ The case of $a_{n} e^{k L} \ll 1$ corresponds to just the zero mode solution.

${ }^{8}$ Since the $\epsilon(n)$ dependence can come into the gravitational potential expression through multiplicative corrections of $1+O(\epsilon)$ through $\chi^{(n)}$ and $m_{n}$, we see that the KK tower summation will be corrected at most by a multiplicative factor of $1+O(\epsilon(1))$.
} 


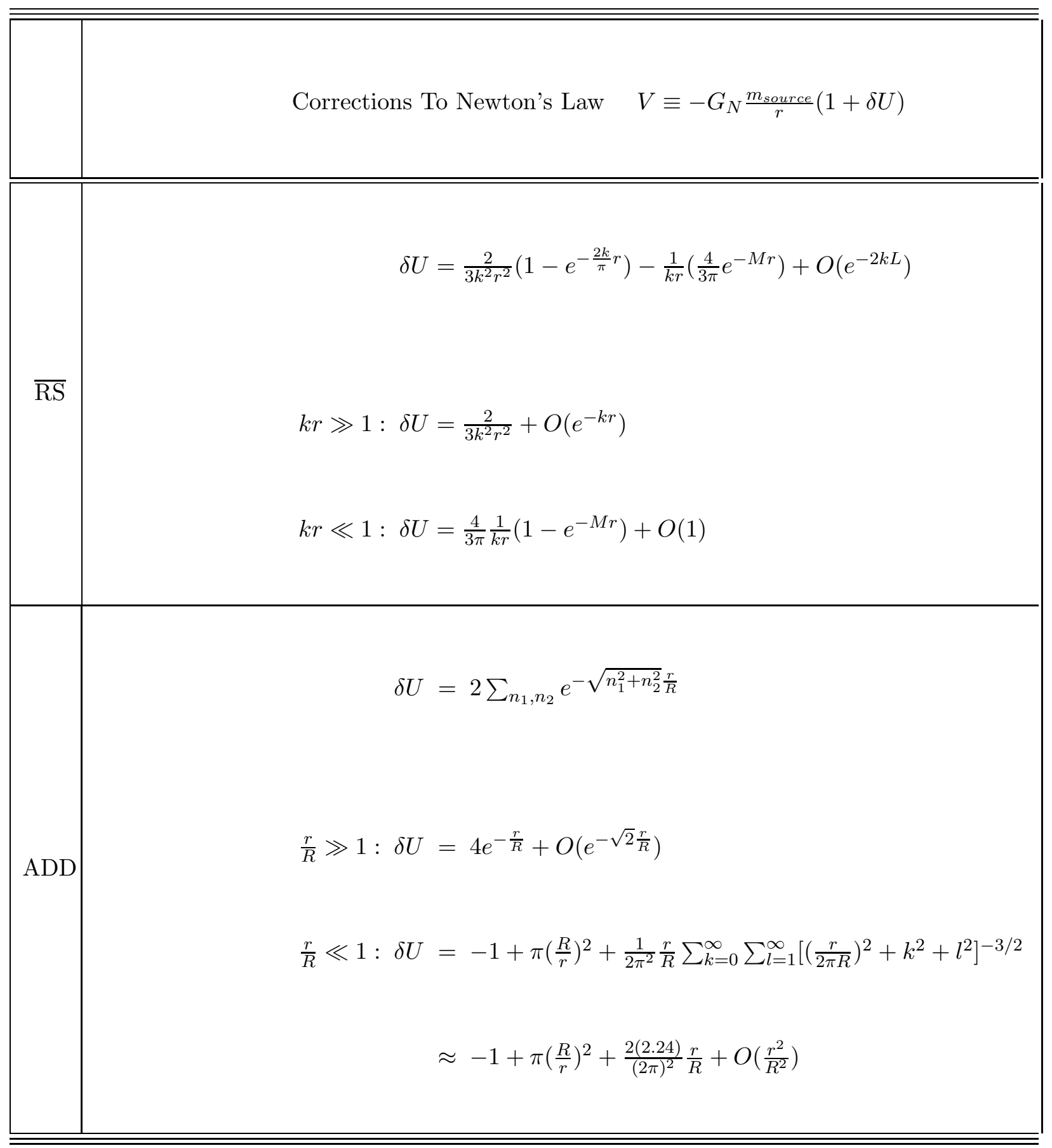

TABLE I. A comparison of the analytic expressions for the corrections to the Newton's Law for the small warping Randall-Sundrum model and the ADD scenario with two extra dimensions. 